\title{
Brain Tumour Segmentation using Level Set Method and Affected Area Calculation
}

\author{
Md. Manik Ahmed ${ }^{1 *}$, Md. Imran Hossain ${ }^{2}$ \\ ${ }^{1}$ Department of Information and Communication Engineering, Pabna University of Science and \\ Technology, Pabna, 6600, BANGLADESH \\ ${ }^{2}$ Assistant Professor, Department of Information and Communication Engineering, Pabna University of \\ Science and Technology, Pabna, 6600, BANGLADESH \\ *Corresponding Contact: \\ Email: kironmanik_pust@yahoo.com
}

\begin{abstract}
Medical image processing is the most important and challenging field now days. MRI image processing is one of the parts of this field. Brain tumour segmentation in magnetic resonance imaging (MRI) has become an emergent research area in the field of medical imaging system. In this paper we proposed a variational level set method and some morphological operation to segment the brain tumour from MRI image by using MATLAB. Actually we describe variational formulation on geometric active contours that forces the level set function at zero level to be close to signed distance function and without re-initialization process. The variational formulation uses energy function and partial diferential equation to evolve the level set function. Tumour shape area is connected component in binary image and calculated this connected area using some properties of morphological operation.
\end{abstract}

Key words

MRI, Segmentation, LSM, Area, Morphological, Matlab

$12 / 15 / 2015$

Source of Support: None, No Conflict of Interest: Declared

This article is is licensed under a Creative Commons Attribution-NonCommercial 4.0 International License.

Attribution-NonCommercial (CC BY-NC) license lets others remix, tweak, and build upon work non-commercially,

and although the new works must also acknowledge \& be non-commercial.

\section{INTRODUCTION}

Tumour is an abnormally increasing growth of tissues in any part of the body. They are of different types and hold different characteristics and treatment (Rana etal). Brain tumor is an abnormal mass of tissue in which cells grow and multiply uncontrollably, seemingly unchecked by the mechanisms that control normal cells. Epilepsy is a brain disorder in which clusters of nerve cells, orneurons, in the brain sometimes signal abnormally. Neurons normally generate electrochemical impulses that act on other neurons, glands, and muscles to produce human thoughts, feelings, and actions. In epilepsy, the normal pattern of neuronal activity becomes disturbed, causing strange sensations, emotions, and behavior or sometimes convulsions (Patil and Bhalchandra). MRI is short for Magnetic Resonance Imaging. It is a procedure used in hospitals to scan patients and determine the 
severity of certain injuries. An MRI machine uses a magnetic field and radio waves to create detailed images of the body (What is MRI?). Magnetic resonance imaging (MRI) is done for many reasons. It is used to find problems such as tumors, bleeding, injury, blood vessel diseases, or infection. MRI also may be done to provide more information about a problem seen on an X-ray, ultrasound scan, or CT scan. Contrast material may be used during MRI to show abnormal tissue more clearly (http://www.webmd.com/).

Segmentation is the division of an image into regions or categories, which correspond to different objects or parts of objects. Every pixel in an image is allocated to one of a number of these categories. Here we used variational level set method to segment brain tumour from MRI images. The variational level set method consist of two energy term first, internal energy term that penalizes the deviation of the level set function from a signed distance function. Second, external energy term that drives the motion of the zero level set toward the desired object boundaries ( $\mathrm{Li}$ etal).The basic idea is to represent geometric active contours as the zero level set of an implicit function defined in higher dimension, usually reffered as the level set function, and to evolve the level set function according to partial differential equation (PDE). These approaches provide some advantages. First, automatically handle the topological changes. Second, Numeric grid point never collide. Third, Natural philosophy for dealing with gray level image. Pre-processing of MRI images is the primary step which is accomplished by applies some filter operation on the input noisy image which is used to enhance the image quality. Finally this image as a input of proposed system.

\section{Traditional LeVel Set Method}

The level set method was developed by the American mathematicians in 1980s Stanley by Osher and James Sethian (Osher and Sethian, 1988). Level Set Method is a numerical technique for tracking interface and shape .Everything now in implicit form we have function define on the plane by moving these function moving the zero level set then we are getting contour. Let we define a function $\Phi$ on the plane and all the point in the plane hold certain equation $\Phi(x, y)=0$.Every point of function $\phi$ inside positive distance and outside negative distance.we have a function $\phi$ all the point that belongs to the curve is zero.A Level Set function $\phi(x, y)$ that is represented a curve in implicit form $C=\{(x, y): \phi(x, y)=0\}$. This curve define this way in moving velocity

$\frac{\partial C}{\partial t}=\mathrm{V} \vec{N}$

Where $\mathrm{V}$ is the velocity and $\mathrm{N}$ is the normal direction which is perpendicular to tangent $\mathrm{T}$. The evolution equation of the level set function Á can be written in the following general form:

$\frac{\partial \phi}{\partial t}=\mathrm{V}|\nabla \phi|$

We move a curve according to velocity $\mathrm{V}$ in a normal direction that obtain by moving $\phi$ up and down whatever this formula tells us that will cut.If time step is 5 do this $t=5$ find the zero level set and we got the curve evaluation. Everything now in implicit form we have function define on the plane and by moving this function we are moving the zero level set we are getting curve evolution for example we are getting active contour. Level Set Method is important for topological changes handle and numerical grid point never collide. 


\section{Proposed Method}

Our proposed Level Set Method uses geometric active contour to segment the image. In image segmentation, active contours are progressive curves that move toward the object boundaries. The main objective is to represent the evolving contour using a signed function, where the zero level corresponds to the actual contour. It is very difficult to keep the evolving level set function as an approximate signed distance function during the evolution. Therefore it is necessary to reshape the level set function to a better form immediately, without changing the zero location. One process to do this is to perform reinitialization, which is a technique for periodically re-initializing the level set function to a signed distance function (Rana etal). The standard re-initialization method to solve the following re-initialization equation:

$\frac{\partial \phi}{\partial t}=\operatorname{sign}\left(\phi_{0}\right)(1-|\nabla \phi|)$

Where, $\phi_{0}$ is the function to be re-initialized, and $\phi$ is the sign function.During evolution re-initialization method has several disadvantage. From the practical viewpoints, the re-initialization can be quite complicated, expensive, and time consumed process. To overcome these, in this paper, we present a new variational level set formulation without re-initialization. It requires intemal energy tenn that penalizes deviations of the level set function from a signed distance function, and an extemal energy term that drives the motion of the zero level set towards the desired image feature (Rana etal). Variational Level Set Formulation consist of three terms, such as penalizing term, edge term, and region term. Penalizing terms is utilized to avoid that periodically re-initializing the level set function to a signed distance function during the evolution. Region terms represent the intensity difference of inside curve and outside curve. Edge terms are the gradient descent flow that minimizes the geodesic length (Yang etal, 2014). The total energy term for curve evolution represented by the following variational formulation

$E=\lambda_{1} P(\phi)+\lambda_{2} L_{g}(\phi)+\lambda_{3} R(\phi)$

where $\lambda_{1}$ and $\lambda_{2}$ are the coefficient of weighted length relative influence of each of the terms on the evolution of the contour. $P(\phi)$ controls the effect of penalizing the deviation of from a signed distance function, $L_{g}(\phi)$ and $R(\phi)$ are respectively the region energy and edge energy term that would drive the motion of the zero level curve of $\phi$.

The Region term derived from the Chan-Vese model for mumford shah energy which is basis on the intensity difference which is formed as follows:

$R(C)=\int_{\text {inside }(C)}\left(I-\mu_{1}\right)^{2} d x d y+\int_{\text {outside }(C)}\left(I-\mu_{2}\right)^{2} d x d y(5)$

where $C$ is the closed contour and $R(C)$ is an energy function, $I(x, y)$ intensity of the image at point $(\mathrm{x}, \mathrm{y})$.

The edge terms is introduced in Geodesic active contour model. It proved that the image segmentation can transfer to find the minimum-length of the zero level set curve of $\phi$ measured in a Riemannian space induced from the image (Yang etal, 2014). Its functional to be minimized is stated as follows: 
$\mathcal{L}_{g}(\phi)=\int_{\Omega} g \delta(\phi)|\nabla \phi| d x d y$

Where $\Omega$ is the open bounded domain. Image gradient is depending on edge indicator function. First we compute the edges we have to do this local computation. Here $g$ is a decreasing function of the Image gradient computing by the edge indicator function that function helps to detect abruptly changes the intensity value area. The edge indicator functions define as follows:

$g(x, y)=\frac{1}{1+\left|\nabla G_{\sigma} * I(x, y)\right|^{2}}$

where $G_{\sigma}$ is the Gaussian kernel with standard deviation $\sigma$. Actually region and edge energy terms drive the motion of the zero level curve of $\phi$ towards the object boundary. It is very difficult to keep the evolving level set function as an approximate signed distance function during the evolution, especially in a neighborhood around the zero level set. It is well known that a signed distance function must satisfy a desirable property of $|\nabla \phi|=1$. Conversely, any function $\phi$ satisfying $|\nabla \phi|=1$ is the signed distance function plus a constant (Li etal). Naturally, we propose the following integral,

$p(\phi)=\int_{\Omega} \frac{1}{2}(|\nabla \phi|-1)^{2} d x d y$

as a metric to characterize how close a function $\phi$ is to a signed distance function in $\Omega \subset$ $\Re$.The first variation of (4) written as $\frac{\partial \varepsilon}{\partial \phi}$ of the functional $\varepsilon$ in which the function $\phi$ that minimizes this functional satisfies the Euler-Lagrange equation $\frac{\partial \varepsilon}{\partial \phi}=0$ here, $\frac{\partial \phi}{\partial t}=-\frac{\partial \varepsilon}{\partial \phi}$

The steepest descent process for minimization of the functional $\varepsilon$ is the following gradient flow:

$\frac{\partial \phi}{\partial t}=\mu\left[\Delta \phi-\operatorname{div}\left(\frac{\nabla \phi}{|\nabla \phi|}\right)\right]+\lambda \delta(\phi) \operatorname{div}\left(g \frac{\nabla \phi}{|\nabla \phi|}\right)+\operatorname{vg} \delta(\phi)$

This gradient flow is the evolution equation of the level set function in the proposed method. The second and the third term in the right hand side of (10) correspond to the gradient flows of the energy functional, $L_{g}(\phi)$ and $\mathrm{R}(\phi)$ respectively, and are responsible of driving the zero level curve towards the object boundaries. During the evolution of $\phi$ according to gradient flow (10) that minimize the functional (4), the zero level curved moved by the external energy. Meanwhile, due to the penalizing effect of the internal energy, the evolving function Á will be automatically maintained as an approximate signed distance function during the evolution according to the evolution (10). Therefore the re-initialization procedure is completely eliminated in the proposed formulation.

\section{IMPLEMENTATION}

Our proposed paper show several stages to segment brain tumour from MRI images, first is pre-processing of given MRI image and after that segmentation the tumour is totally detected.

Stages are following: 


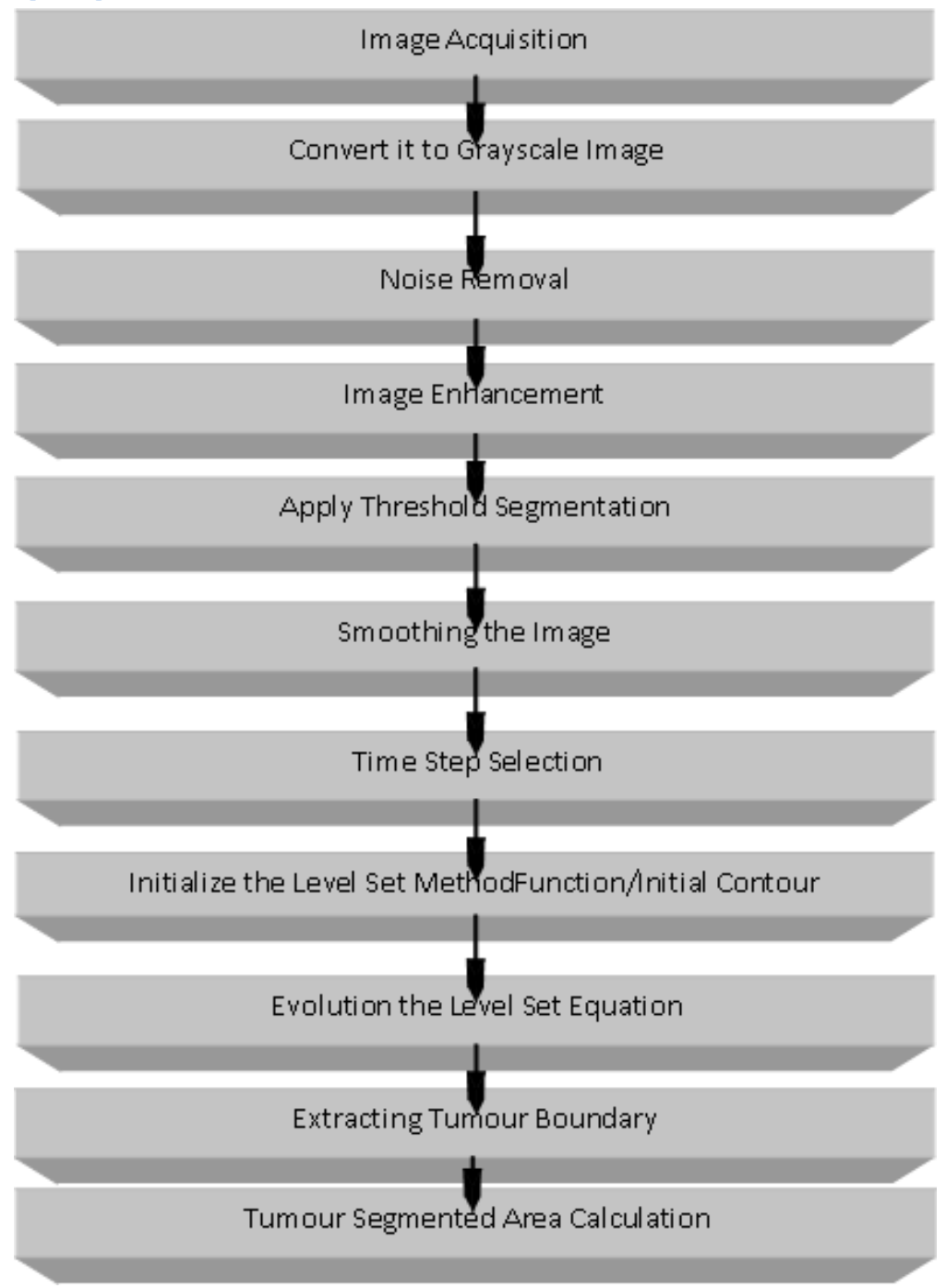

Figure 1: Total Outline of the Proposed System

The segmentation processes is implemented as depicted in Figure (1) above. In this thesis paper, we wanted to segment brain tumour using Level set evolution method. To reach destination our first stage is image acquisition. Images are obtained from MRI scan on brain which images format are portable network graphics (.png). For proper utilization of these images we need to convert it to grayscale images which are data matrix whose value represents shades of gray. The intensity value of gray scale matrix are integer values in the range [0 255]. The patient who suffers brain tumour and we get the brain tumour image from the MRI scanner machine of this patient. In this image shows some region of having high intensity value. Our proposed algorithm needs grayscale image because each pixel of grayscale image is a 8 bit number and it takes value from 0 to 255 which is very useful for our proposed algorithm, where affected area of brain tumour having high intensity value and we need to fixed this area as some intensity value. MRI images have some noise so next step is to remove noise using some filter operation. 
Here are various kinds of filter available in image processing and they are different characteristics and used for removing noise from the image. In this paper we used spatial noise filter for removing image noise.

There are two kinds of filters:

a. linear filters;

b. nonlinear filters.

For enhancing image we use two non linear filter which are:

a. high pass filter;

b. median filter

c. Laplacian filter

Image Enhancement play a vital role for image segmentation. If we give the Enhanced images as input, we get the better result. More enhanced image more better result. Enhancement will give the result more projected edges and a sharpened image is obtained, noise will be reduced thus reducing the blurring effect from the image. This process improving the quality of the overall image. Edge detection will lead to finding the exact location and size of tumor. For enhancing MRI images, In this paper we have been applied spatial noise filter like high pass filter, median filter, Laplacian filter e.t.c for removing image noise. These filters removes blurring effect from image and highlight edges. Laplacian and High Pass filters are sharpening filter. After applying Laplacian filter to the image, a new image is obtained highlighting edges and other discontinuities. The output image obtained from the laplacian filter give much better result than other filter. After getting the enhanced image, the process of detection of exact location and size of tumour.

To get better result for Tumour Segmentation we need to provide enhanced image (Fig.5) as input then threshold the images which eliminate the intensity value of each pixel less than 200. Then smoothing the image by Gaussian convolution. In implementing the proposed system, the time step $\tau$ can be chosen significantly larger than the time step used in the traditional level set methods .we selected time step $\tau$ in our experiment 2.5 and getting final contour after 800 iteration which brain tumour is detected. We have also tried large range of time step in our experiment such as 1 to 100.Also we use time step $\tau$ in our experiment 5 and getting final contour after 600 iteration. From our experiments, we have found that the time step $\tau$ and the coefficient $\mu$ of internal (penalizing) energy term must satisfy $\tau \mu<\frac{1}{4}$ in the difference scheme. In traditional level set methods, it is necessary to initialize the level set function $\phi$ as a signed distance function $\phi_{0}$.

Suppose $\phi_{0}$ is the initial function, let region $\Omega_{0}$ be a subset in the image domain $\Omega$, and $\partial \Omega_{0}$ be all the points on the boundaries $\phi_{0}$. Then $\phi_{0}$ initialize as:

$\phi_{0}(x, y)=\left\{\begin{array}{c}-\mathrm{D}_{0}(x, y) \in \Omega_{0}-\partial \Omega_{0} \\ 0 \quad(x, y) \in \partial \Omega_{0} \\ \mathrm{D}_{0} \quad \Omega-\Omega_{0}\end{array}\right.$

In this experiment $\phi_{0}$ function is defined by (15) with $\mathrm{D}=4$ and this initial function $\phi$ only takes three value $-4,0$ and 4 .In our segmentation process we use some parameter sigma $=1.5$, lambda $=5$, alfa $=1.02$ and epsilon of the smooth dirac function default value 1.5.Finaly we have calculated Tumour Segmented Area calculated where Area is the actual number of pixels in the region. We have calculated this segmented Area or actual number of pixel of our image is 1957. We got the value of Tumour Segmented Area from our input image. 


\section{EXPERIMENTAL RESULT}

Firstly, we use a brain MRI image a brain tumour is presence. Next figure show the images as an output, i.e grayscale image,high pass filtered image, laplacian filtered image, enhanced MRI image which are more smooth and sharpened than input noisy image. In Fig. (7) the input image and corresponding Fig. (9) shows the evolution level set curve for separate number of iteration. We observe a segmented brain tumour in final contour. Fig.(10) shows the finaly segmented Tumour Region marking by red line and Fig. (11) shows the tumour area or actual pixel of Fig.(10) give the value is 1957. This segmentation system is very useful for medical diagnosis system.

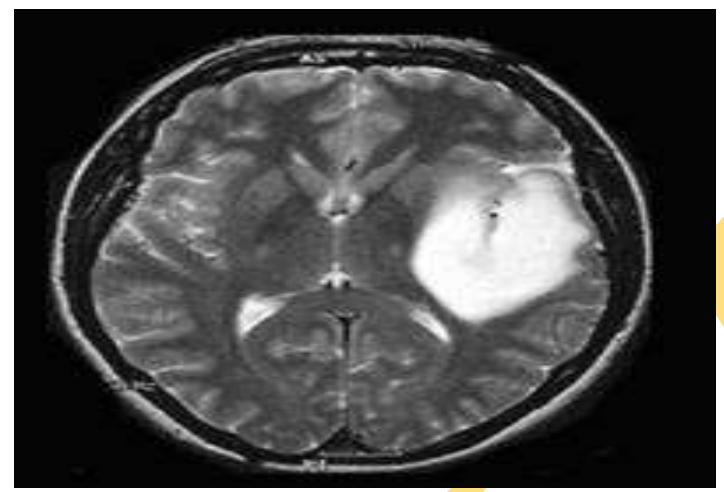

Figure 2: Input noisy image of brain

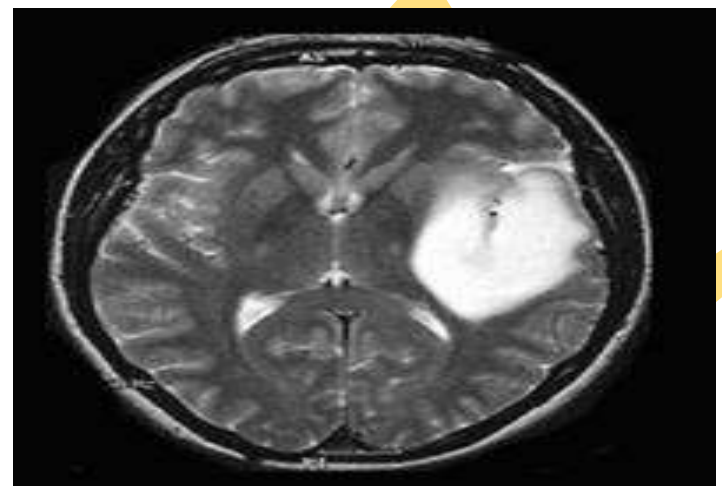

Figure3: Grayscale image of the figure 2

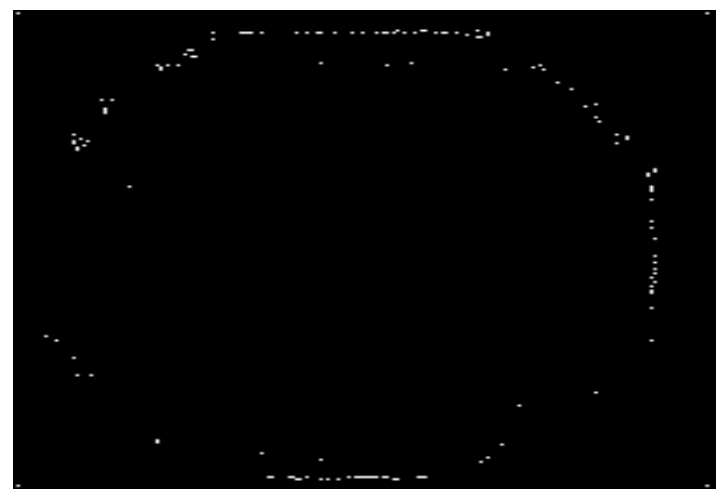

Figure 4: High pass filter output of figure 3 


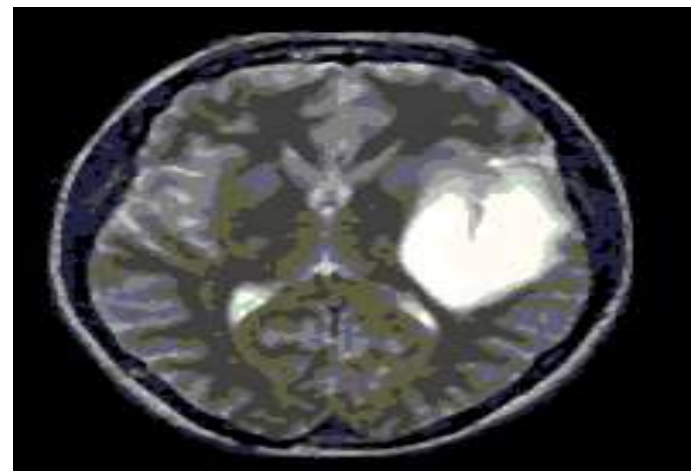

Figure 5: Median filter output image of figure 3

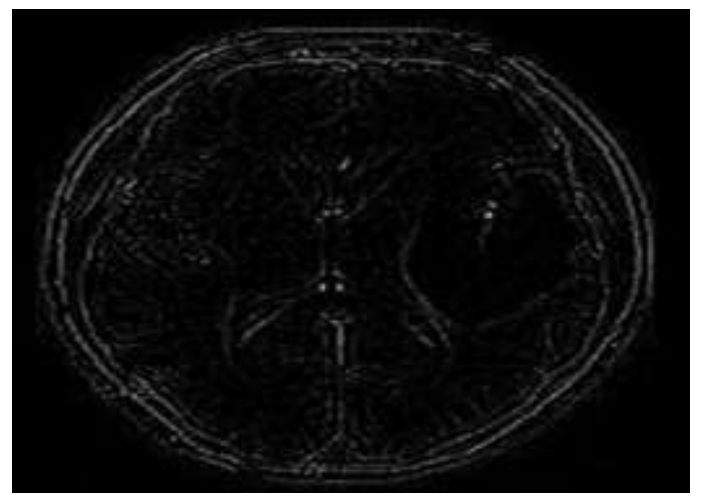

Figure 6: Edge detected image of figure 5

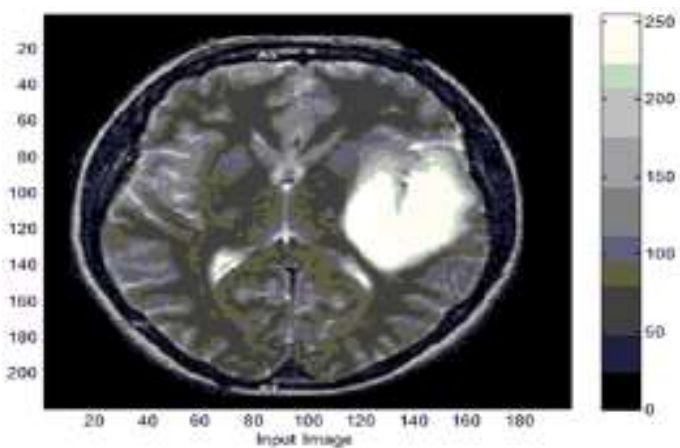

Figure 7: Input image of the proposed system with color bar

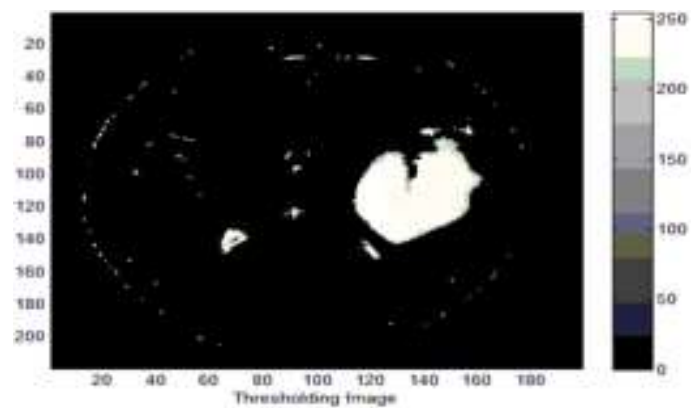

Figure 8: Thresholding image of figure 7 with color bar 


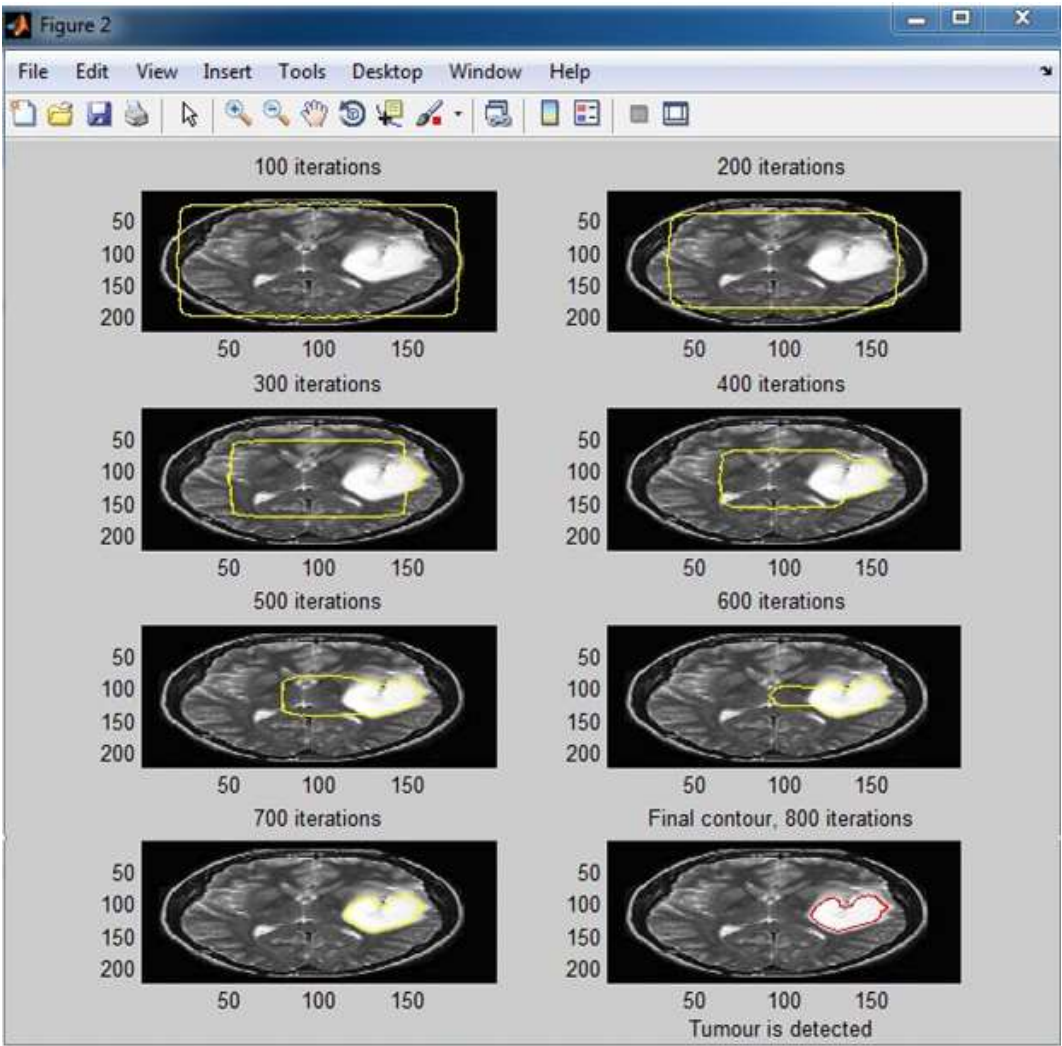

Figure 9: Output of the proposed method with different iteration and the final contour (Detected tumour)

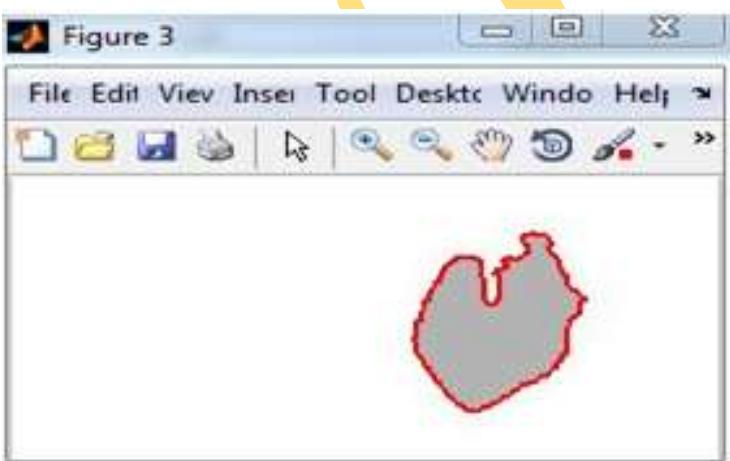

Figure 10: Observing the final Tumour Segmented Area

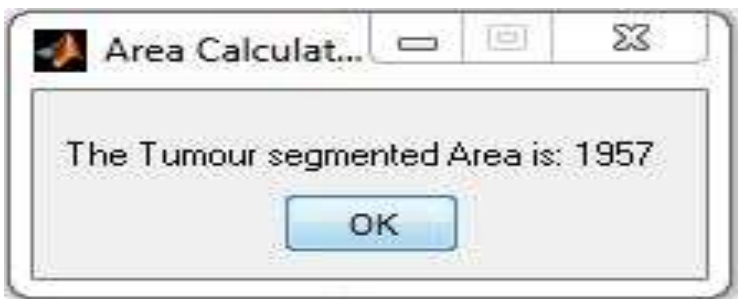

Figure.11.Tumour Segmented Area calculated from figure.11 


\section{Conclusions}

In this paper we use variational formulation level set algorithm without re-initialization. It is easy to implement and more efficient than traditional level set method. To speed up curve evolution we should be used larger time step. Also this paper gives more information about brain tumor detection and segmentation use this algorithm. The marked area is segmented and this procedure removes the overhead of the radiologist and helps them in diagnosis, the treatment procedure and state of the tumor growth observation and monitoring.

\section{REFERENCES}

Chunming Li, Chenyang Xu, Changfeng Gui, and Martin D. Fox, "Level Set Evolution Without Reinitialization: A New Variational Formulation".

Hongzhe Yang, Lihui Zhao, and Songyuan Tang. "Brain Tumor Segmentation Using Geodesic Region-based Level Set without Re-initialization", "International Journal of Signal Processing, Image Processing and Pattern Recognition", Vol.7, No.1 (2014), pp.213-224.

Information and Resources, "http://www.webmd.com/a-to-z-guides/magnetic-resonance-imagingmri".

Rachana Rana, H.S. Bhdauria, Annapuma Singh, "Brain Tumour Extraction from MRI Images Using Bounding-Box with Level Set Method".

Rajesh C. Patil and Dr. A. S. Bhalchandra, "Brain Tumour Extraction from MRI Images Using MATLAB", "International Journal of Electronics, Communication \& Soft Computing Science and Engineering", ISSN: 2277-9477, Volume 2, Issue 1.

S. Osher, J. A. Sethian, "Fronts propagating with curvaturedependent speed: algorithms based on Hamilton-Jacobi formulations", J. Comp. Phys., vol. 79, pp. 12-49, 1988.

What is MRI? How does MRI work?, http://www.medicalnewstoday.com/articles/146309.php

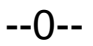

Online Archive Link: $\underline{\text { https://abc.us.org/ojs/index.php/ei/issue/archive }}$ 